\title{
In-Situ Lithiation/Delithiation Observation of Individual Amorphous Si Nanorods
}

\author{
Hessam Ghassemi ${ }^{*}$, Ming $\mathrm{Au}^{2}$, Reza Shahbazian-Yassar ${ }^{1}$
}

${ }^{1}$ Department of Mechanical Engineering-Engineering Mechanics, Michigan Technological University, 1400 Townsend Dr., Houghton, MI 49931

${ }^{2}$ Savannah River National Laboratory, Aiken, SC

Lithium ion batteries are considered as the future power source for the electric vehicles and other portable electronic devices. The anode material properties, such as energy storage and its cycle life, basically limit the high efficiency of a lithium ion battery [1]. Silicon is the most attractive material due to its low discharge potential and the highest theoretical charge capacity, $4200 \mathrm{mAh} / \mathrm{g}$. However, insertion/extraction of Li into the Si structure expands/shrinks its volume by $400 \%$, which reduces the capacity as a result of pulverization, over time. Recently, it was shown that Si nanorods can prevent pulverization by lateral expansion [2]. No real-time study, yet, shows how the nanorods accommodate the strain upon lithiation/delithiation. Here, for the first time, we investigate the lithiation of individual amorphous Si nanorods using a unique in-situ transmission electron microscopy (TEM) technique. Two different diffusion paths, longitudinal and radial, were examined and the formation of $\mathrm{Li}_{\mathrm{x}} \mathrm{Si}$ phase was confirmed by diffraction pattern studies that are in good agreement with our ex-situ lithiation results. The induced-strain as a result of lithiation was measured around $270 \%$ and no pulverization in individual NRs was observed. Our results prove the fact that NR can accommodate the large strain by lateral expansion.

Individual Si NRs were attached on an Au wire by light mechanical scratching on the as grown samples. Figure 1a depicts the in-situ lithiation set-up where a drop of ionic liquid (IL) is placed on the STM tip and individual Si NRs are placed on the gold wire. The applied bias voltage flows the IL on the NR from STM side to the gold wire, as shown and indicated in Figure 1b. High-resolution TEM imaging and electron diffraction pattern were collected to characterize the formation of different phases during the lithiation of different individual Si NRs.

Figure 2 compares lithiation process of different individual Si NRs with two different lithium concentrations in the IL. Series I shows the insertion of Li into the Si NR from low concentration of IL. The experiment was carried out for $30 \mathrm{~min}$. We, then, examined the effect of Li concentration in the IL as shown in Figure 2 series II. At higher concentration of Li, our observations show that the formation

1 Uday Kasavajjula et al. Journal of Power Sources 163, (2007), 1003-1039

2 Candacek Chan et al. Nature Nanotechnology 3, (2008) 31-35 
and growth of $\mathrm{Li}_{\mathrm{x}} \mathrm{Si}$ phase was expedited. As shown in Figure $2 \mathrm{~g}$, formation of $\mathrm{Li}_{\mathrm{x}} \mathrm{Si}$ particles starts just after 3 min of lithiation as a result of higher Li ion concentration. Following the lithiation process, after $10 \mathrm{~min}$ as shown in figure $2 \mathrm{~h}$, particles grow to $\sim 6 \mathrm{~nm}$ which is larger than the $4 \mathrm{~nm}$ particles formed in the low concentration case as shown in Figure 2d, for 30 min of lithiation.

Figure 3a shows an individual Si NR placed on the high-concentration IL to make the is-situ experiment as similar as possible to the real-time ex-situ charging experiment. After lithiation the lateral expansion of NR was measured to be $70 \mathrm{~nm}$ which is $270 \%$ expansion compared to the initial. This result is in strong agreement with real-time battery working condition where the NRs are actually dipped into the electrolyte and diffusion path is radial. As expected and shown by the ex-situ studies, no pulverization was captured.
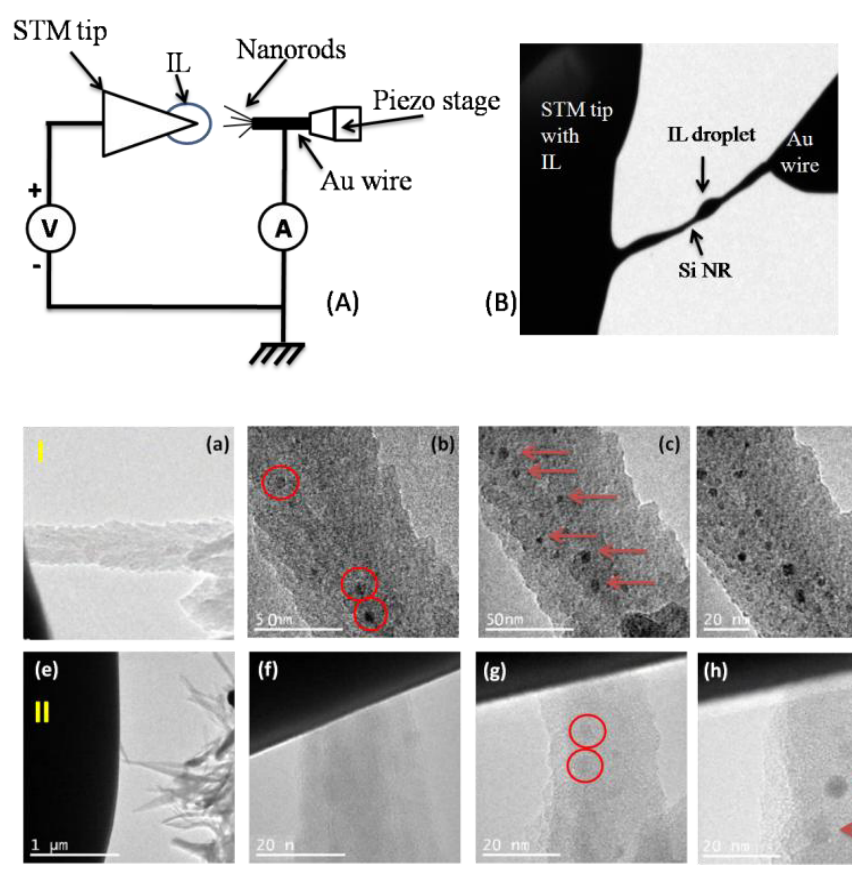

Figure 1 (a) schematic of the STM holder experimental setup. As the STM tip is positively biased, IL flows toward the nanorods and Li ions diffuse into nanorods. (b) low-magnification image during the lithiation experiment. The arrow indicates a droplet as the IL flows on the Si NR (the other arrow) from the STM side to the Au wire.

Figure 2.Series (I a-d) represent sequence of insertion of Li from low concentration IL into the Si NR during 30 min. (a) shows low magnification of individual Si NR connected to IL (dark triangle on left). (b) Red circles indicate the formation of $\mathrm{Li}_{X} \mathrm{Si}$ phase after 10min. (c) and (d) show the growth and formation of new particles as the lithiation process continues, $20 \mathrm{~min}$ and $30 \mathrm{~min}$, respectively. Series (II e-f) show sequence of

lithiation at higher Li concentration during $10 \mathrm{~min}$. (e) and (f) show low- and high-magnification images of individual Si NR attached to IL. After 3 min, as shown in (g), the formation of new phase was detected. And (h), growth and formation of $\mathrm{Li}_{\mathrm{x}} \mathrm{Si}$ particles are indicated with arrows, after $10 \mathrm{~min}$.
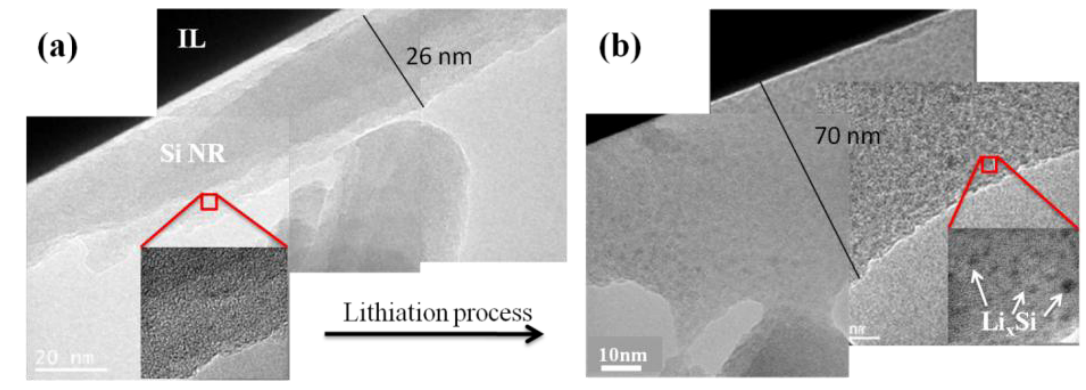

image of $\mathrm{Li}_{\mathrm{x}} \mathrm{Si}$ particles formed as a result of lithiation.
Figure 3 (a) individual Si NR in contact with IL from the side. The thickness of the NR was measured to be $26 \mathrm{~nm}$ before the lithiation and high-resolution image (inset) shows amorphous structure. (b) After lithiation process the diameter of NR expands to $70 \mathrm{~nm}$ and is dipped into the IL, inset show high-resolution 